\title{
Analysis of P2P File Sharing Network's Credit System for Fairness Management
}

\author{
Yunzhao Li \\ Electrical and Computer Engineering \\ Kansas State University \\ yzli@ksu.edu
}

\begin{abstract}
Fairness is an important management issue for peer-to-peer file sharing systems. In this paper, we study the credit system of the P2P file sharing network eMule (http://www.emule-project.net) through a simple queueing network model. Numerical analysis and experimental results show that this local credit strategy could effectively deal with free riders and provide fairness for the system during a single file exchange. Using this model, we also investigate different management strategies for dealing with the newcomer fairness issue. We propose a simple, private history-based scheme to balance the fairness between two types of newcomers.
\end{abstract}

\section{INTRODUCTION}

The recent popularity and success of peer-to-peer (P2P) file sharing has established its importance while also contributing a majority of the traffic on the Internet. In contrast to the traditional client-server content distribution system, every member of a P2P file sharing network has an equivalent role. Not only can each peer download from other peers, but it is also responsible for uploading content as a server. This often results in fairness issues as many peers called free riders may only want to download without uploading or sharing their own content. This paper evaluates the performance of two types of peers that are subjected to a fairness management policy which gives download priority to users who also upload their content to the network.

The distributed architecture of a P2P system does not easily lend itself to control free riders in order to maintain fairness. In fact, as a somewhat autonomous system, the file sharing performance of a P2P network greatly depends on each peer's cooperation. A peer should be willing to voluntarily donate resources in exchange for content that it would like from other peers. However, selfish peers exist who benefit from other peers' contribution yet refuse to offer in exchange their own resources.

Such selfish behavior will result in the eventual collapse of the whole system. In an attempt to dissuade this behavior, various incentive management strategies are introduced to current $\mathrm{P} 2 \mathrm{P}$ file sharing networks to reward general peers which share their information and penalize free riders. For example, a BitTorrent client prefers to allocate upload bandwidth to peers who send data to it with a rate-based titfor-tat fairness policy [1]. Another fairness control strategy is to build a global trust management system, which could help peers choose their neighbors based on different trust levels.

\author{
Don Gruenbacher \\ Electrical and Computer Engineering \\ Kansas State University \\ grue@ksu.edu
}

For example, in [2], the global reputation scores of all nodes in the current unstructured P2P network are collected, calculated, and then distributed in the whole system.

Unlike the previous fairness policies, the eMule P2P file sharing application adopts a simple local trust system called a credit system to encourage peers to exchange information while restricting free riders. The eMule network is a local reputation system that only allows credit to be exchanged between the uploader and its downloader. This is different from a global reputation system that allows credit to be exchanged among all peers.

While almost all of the current $\mathrm{P} 2 \mathrm{P}$ research contributes to multiple aspects of BitTorrent, eMule's fairness issue is lightly addressed even though it significantly impacts whole system performance. Currently, global and public historybased reputation approaches can be regarded as possible solutions for P2P network's fairness management. However, compared to the complexity of the global system, the simple, local, and private history-based approach also needs to be carefully investigated. Therefore, the work presented here evaluates eMule's local and private history-based credit system for maintaining fairness. The primary contributions of this paper are:

1) a simple queueing network model is developed to investigate the impact of credit on system fairness; both the numerical analysis and the experiments in the real world illustrate that even if the incentive algorithm is local-based, it can still deal with free riders and provide fairness during a single file exchange when compared to BitTorrent's "TFT" incentive algorithm;

2) our model is used to compare two different types of credit strategies for providing fairness to the newcomer. A simple, long-duration, and private history-based credit scheme is proposed which will better reward the generous newcomer while limiting the selfish free rider.

The paper is organized as follows. Section 2 discusses important related work. In section 3, a queueing network model is presented to study the fairness of eMule. The numerical analysis and corresponding experiment results are shown in section 4. Section 5 discusses the newcomer management issue and an improved fairness design is presented for providing fairness to newcomers, followed by summary and future work in section 6 . 


\section{RELATED WORK}

There are a large number of publications related to various aspects of peer-to-peer file sharing such as performance, fairness, and security. The work in this paper is motivated by [3] and [4]. In [3], a mathematical model is developed for studying BitTorrent's performance, and the authors find the distribution of download peers into the system takes the form of an asymmetric U-shaped curve. This means there are more peers blocked at the beginning and end segments of the download process than peers at the other download time period. In [4], a general stochastic analytic framework for incentive-based file-swarming research is proposed, and the first-chunk problem is also shown in the authors' analytical bound and simulation result. The first-chunk problem is related to how the system manages newcomers for which a more detail discussion will be given in the following section.

Other works are also given attention to P2P system fairness. In [5] and [6], game theory is used to investigate the relationship among peers. The fairness policy of a current peer-to-peer file sharing system such as BitTorrent is shown to not be robust in [7], and the free rider could obtain a higher download rate than a tit-for-tat compliant client [8]. In [2] and [9], global reputation approaches are suggested for dealing with free riders and malicious peers. However, reputation is always vulnerable when the free rider repeatedly changes its ID for additional benefit, or more than one free rider work together as a coalition [10]. Furthermore, because of the complexity of implementation, the global reputation approach hasn't been popularly employed from existing P2P file sharing systems in the real world.

Although BitTorrent has gathered more attention than eMule in the research community, there are a number of papers that do address eMule's performance. In [11], a fluid model is developed for the analysis of a system like eMule, and an optimal upload strategy is given. In [12], the authors investigate file diffusion through an epidemic method, and the influence from corrupted files is shown. The projects in both [13] and [14] respectively measure P2P networks such as eMule from both the client viewpoint and the ISP's viewpoint. The measurements include eMule's traffic characterization, the whole system's capacity, and sharing files' distribution.

\section{QueUEING NeTwORK MODEL}

\section{A. The eMule P2P File Sharing System}

As one of the most popular file sharing systems, eMule averages more than 2 million peers in the system during October 2005 [15]. It uses a hybrid architecture, which first obtains online sources information for its expected content, then employs multiple sources in downloading by dividing the whole file into equal-sized pieces called chunks. Through this scheme, more servers appear in the system at the same time to really enhance the system capacity.
eMule employs an incentive fairness algorithm based on a local private history credit record to encourage uploading, which means the credit is used to reward a peers' sharing behavior and provide benefit for future downloading. For example, if peer A uploades a resource to peer B, peer B will give some credit to peer $\mathrm{A}$, and this credit record is only held by peer B. When peer A later wants to download content from peer B, it will be given higher priority service from peer B than other neighbors of $\mathrm{B}$ without credit. Because credit rewards can only be exchanged between the downloader and its directed uploader, the credit information is not spread among other peers. Thus eMule's credit is a local private history-based credit system and not a global public historybased system like some kinds of social reputation networks.

Compared with BitTorrent's use of a simple accept or reject policy for each requester which is unfair for low-bandwidth users, eMule has implemented a complicated priority queue component to cache various requests independent of the customer's upload bandwidth. The positions of peers in an eMule uploading queue are determined by their past credit from the service provider. Because the entire download process is chunk-based, and each chunk can be independently exchanged among peers, eMule's credit operations naturally follow this exchange process. Moreover, the unconsumed credit will reside with peers for a long time period (several months) [17], and this credit can also be used for future file exchanges.

\section{B. Mathematical Model}

We extend the established model in [3] [4] which only focus on the general behavior for P2P file sharing, and distinguish the peers into two types within the model: the general peer who obeys the incentive rule to download and upload, and the free rider who is the extreme malicious peer only downloading from others and refusing to contribute. Most general peers possess positive credit due to their incessant sharing behavior, and as long as a peer has credit, the amount of credit does not differentiate one peer from another.

If a shared file has $K$ chunks, the peer's entire downloading progress could be described by the completion of a task for each chunk downloaded. The file will be completely downloaded at the completion of the $K^{\text {th }}$ task. A simple tandem queueing network that uses a total of $K$ service workstations is suitable for modeling this process. The service station $S_{1}$ can be explained as the location where the peer obtains its first chunk, and the service station $S_{2}$ is the location where the peer obtains its second chunk, and so on. However, the order of the chunk in the whole file does not matter. For example, when a new peer arrives at the system to download a single file, it will send a request from its first service station $S_{1}$ and participate in the uploading queue for $S_{l}$. The peer's queue position is decided by its credit previously earned from its current service providers. After a certain waiting time, it receives the uploading bandwidth and obtains its first chunk. As a result, it could provide upload service for other peers and 


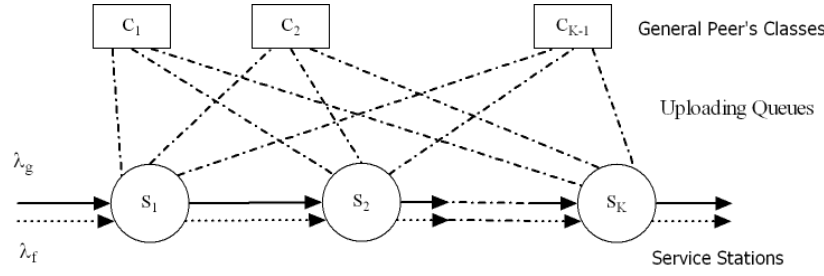

Figure 1. Queueing Network Model. The general peer's arrival rate is $\lambda_{g}$, and the free rider's is $\lambda_{f}$. the general peers with chunks can provide upload bandwidth at classes $C 1, C 2, \ldots$, and after downloading chunks at stations successively, the peers leave the system. The dash lines between the classes and the stations show the distribution of uploading bandwidth.

earn credit $B$ from them. When it begins to download the next chunk, the received credit $\mathrm{B}$ might help it obtain a high priority queue position from the uploading queues. Continuing with this process, earning credit from sharing and consuming credit from downloading, it will finally finish the entire file downloading process (Fig. 1).

It is worth noting that in our model, each station's service providers come from all the general peers having at least one chunk. Thus, we sort these peers into $K-1$ classes: class $C_{l}$ representing peers having one chunk through class $C_{K-1}$ which represents peers having $K-1$ chunks. Two uploading queues exist at each class, each with a different priority. During each time slot, the first peer in the service station's high priority queue will be serviced at the station and complete one chunk's downloading. If no peer exists in the high priority queue, the first peer in the low priority queue will be serviced. A general peer has a probability of joining the high priority queue if the server has previously received its service and assigned some credit to it, while a free rider will always stay in the low priority queue. Consequently, we map the service stations' downloading queues to these classes of the peer's high priority queue and low priority queue which the dashed lines represent between classes and service stations in Fig. 1. Based on the $\mathrm{P} 2 \mathrm{P}$ file sharing system's policy, general peers with $i$ chunks always have two positions in our model: one is in the class $C_{i}$ to provide uploading service, the other is at service station $S_{i+1}$ 's downloading queue for waiting for the service.

Table 1 shows our model parameters. Following the general assumption of the $\mathrm{P} 2 \mathrm{P}$ arrival process used in [3] and [4], we use a Poisson process to model the general peer's arrival rate $\lambda_{g}$ as well as the free rider's arrival rate $\lambda_{f}$. We assume that both types of peers have an equal upload rate $\mu$ and an equal download rate $v$. In P2P networks like eMule, a peer can obtain other peer information through different search methods such as index servers, source exchanges, and a Kademlia DHT network [17]. Thus, the assumption that peers know the other online peers' connection information is rational. For simplicity reasons, it is also assumed that each peer cannot download the next chunk until it completes downloading the current chunk. In our model, peers are assumed to stay in the system during the download. However, they will leave the system immediately after receiving the complete file.

Table 1: Queueing Network Model Parameters

\begin{tabular}{|c|c|}
\hline Parameters & Meaning \\
\hline$\lambda_{g}$ & the arrival rate of general peers \\
\hline$\lambda_{f}$ & the arrival rate of free riders \\
\hline$G_{i}(t)$ & $\begin{array}{l}\text { the number of general peers having } i-1 \text { chunks at service } \\
\text { staion } i\end{array}$ \\
\hline$F_{i}(t)$ & $\begin{array}{l}\text { the number of free riders having } i-1 \text { chunks at service } \\
\text { station } i\end{array}$ \\
\hline$K$ & $\begin{array}{l}\text { total number of chunks in a file, it is equal to the total } \\
\text { number of service stations in the queueing network }\end{array}$ \\
\hline$\mu$ & peer's average upload rate \\
\hline$v$ & peer's average download rate \\
\hline$S_{i}$ & service station $i$ \\
\hline$C_{i}$ & general peer's class $i$ \\
\hline$H_{j}$ & the high priority queue size of class $j$ \\
\hline$L_{j}$ & the low priority queue size of class $j$ \\
\hline$R_{g}\left(S_{i}, S_{i+1}\right)$ & $\begin{array}{l}\text { general peers' transfer rate from service station } S_{i} \text { to } \\
\text { service station } S_{i+1}\end{array}$ \\
\hline$R_{f}\left(S_{i}, S_{i+1}\right)$ & $\begin{array}{l}\text { free riders' transfer rate from service station } S_{i} \text { to service } \\
\text { station } S_{i+1}\end{array}$ \\
\hline
\end{tabular}

Because of its limited participation time and few shareable chunks, the general peer may only earn low credit at the beginning of downloading. Therefore, it has a low probability of obtaining better service from the beginning service stations. The effect of previously earned, long-term credit is ignored and will be explained in section IV. This will rapidly change as its sojourn time in the system increases and it has more chunks to share. Thus we adopt the exponential utility function to represent the probability $P_{r}(i)$ that general peers at service station $S_{i}$ can enjoy the credit benefit:

$$
\operatorname{Pr}(i)=1-e^{-\beta(i-1)} \quad 1 \leq \mathrm{i} \leq \mathrm{K} \quad 0 \leq \beta \leq 1 .
$$

The distribution of requests among all classes uploading queues is now considered. Following [3], peers are considered that possess one or more chunks and have identified missing chunks at one or more peers. The probability of joining class $C_{j}$ 's uploading queue is calculated as follows: if peer A has $i$ chunks and requests the remaining $K-i$ chunks from peer B at class $C_{j}$, the probability that peer B contributes to peer $\mathrm{A}$ is:

$$
P(i, j)=\frac{K-i}{K} \frac{j}{K} .
$$

Because each peer is assumed to know the file-swarm's connection information, the normalized probability $P_{b}(i, j)$ that 
it currently owns $i$ chunks and belongs to class $C_{j}$ 's uploading queue is:

$$
P_{b}(i, j)=\frac{\frac{K-i}{K} \frac{j}{K}}{\sum_{j=1}^{K-1} \frac{K-i}{K} \frac{j}{K}}
$$

Now, we can respectively estimate the length of each class $C_{j}$ 's uploading queue for both the high priority queue $H_{j}$ and the low priority queue $L_{j}$ :

$$
\begin{aligned}
& H_{j}=\sum_{i=1}^{K} G_{i}(t) P_{b}(i, j) P_{\mathrm{r}}(i) . \\
& L_{j}=\sum_{i=1}^{K}\left(F_{i}(t) P_{b}(i, j)+G_{i}(t) P_{b}(i, j)\left(1-P_{\mathrm{r}}(i)\right)\right) .
\end{aligned}
$$

The upload rate $\mu_{j}$ of each class $j$ is respectively assigned to both the high priority and low priority uploading queue with percentages of $P_{h}(j)$ and $P_{l}(j)$. Assuming peers in the low priority queue need to wait until all general peers in the high priority queue complete their downloading jobs, their received rates are $1 / H_{j}$. These percentages are:

$$
\begin{aligned}
& P_{l}(j)=1-P_{f}(j)=\frac{1}{H_{j}+1} . \\
& P_{h}(j)=\frac{H_{j}}{H_{j}+1} .
\end{aligned}
$$

Combining the above equations, we get the transfer rate formulas of two types of peers:

(1) $R_{g 1}$ : general peers' transfer rate from service station $S_{i}$ to service station $S_{i+1}$ when they use their credit to join highpriority queues. It is the summation of the upload bandwidth obtained from general peers at class $C_{1}$ to general peers at class $C_{k-1}$.

$$
R_{g 1}\left(S_{i}, S_{i+1}\right)=\sum_{j=2}^{K} \frac{\mu}{v} G_{j}(t) P_{h}(j) \frac{G_{i}(t) P_{b}(i, j) \operatorname{Pr}(i)}{H_{j}} .
$$

(2) $R_{g 2}$ : general peers' transfer rate from service station $S_{i}$ to service station $S_{i+1}$ when they can't use their credit and join the low-priority queues.

$$
R_{g 2}\left(S_{i}, S_{i+1}\right)=\sum_{j=2}^{K} \frac{\mu}{v} G_{j}(t) P l(j) \frac{G_{i}(t) P_{b}(i, j)(1-\operatorname{Pr}(i))}{L_{j}} .
$$

(3) $R_{g}$ : the total transfer rate of general peers from service station $S_{i}$ to service station $S_{i+1}$ is:

$$
\begin{aligned}
& R_{g}\left(S_{i}, S_{i+1}\right)=R_{g 1}\left(S_{i}, S_{i+1}\right)+R_{g 2}\left(S_{i}, S_{i+1}\right) . \\
& R_{g}\left(S_{1}\right)=\lambda_{g}
\end{aligned}
$$

(4) $R_{f}$ : free riders' transfer rate from service station $S_{i}$ to service station $S_{i+1}$ is:

$$
\begin{aligned}
& R_{f}\left(S_{i}, S_{i+1}\right)=\sum_{j=2}^{K} \frac{\mu}{\nu} G_{j}(t) P l(j) \frac{F_{i}(t) P_{b}(i, j)}{L_{j}} . \\
& R_{f}\left(S_{1}\right)=\lambda_{f}
\end{aligned}
$$

The departure rate of station $S_{i}$ is equal to the arrival rate of the state $S_{i+1}$ followed the Markov Chain property [16].

\section{RESULTS AND DISCUSSION}

\section{A. Numerical Analysis}

The mathematical model is intended to answer the question: is the credit system for eMule P2P systems suitable for maintaining system fairness and reducing the damage from free riders? For our model, the time slot is the time to finish one chunk downloading. We choose the value of $\mathrm{K}=25$ to represent the general RMVB file (about $200 \mathrm{MB}$ size in the P2P file sharing system, and a chunk's size of 9.28MB). The value of $\beta=0.1$ is used to calculate the probability $P_{c}(i)$. The ratio of peer's download rate $v$ to its upload rate $\mu$ is chosen as 0.1 , which is the typical value for the current ADSL technique [19]. It is reasonable to believe that the majority of the peers in the P2P system are general peers, while it is still desirable to know the ability of credit to maintain fairness when different proportions of free riders are in the system. Thus we randomly generate the peers' arrival rates through the Poisson distribution, and varied the ratio of general peers' arrival rate to free riders' $\lambda_{\mathrm{g}} / \lambda_{\mathrm{f}}$ from $10,2,1$, to 0.5 . Following the rule for the transfer rate between adjacent states (the state $S_{i}$ 's output rate is equal to the input rate of $S_{i+1}$ ), a numerical analysis is run for the equations 10, 11 in our model until the system reached a stable state.

Fig. 2(a) and Fig. 2(b) plot the variation tendency of the total population of the general peers and the free riders completing their jobs, respectively. Fig. 2(c) shows the ratio of finished general peers to finished free riders over time. The initial value of the numerical analysis introduces some fluctuation at the beginning period of Fig. 2(c). However, when the system reaches steady state, the number of finished general peers is much larger than the number of finished free riders, even when the general peer arrival rate is significantly smaller than the free rider arrival rate. The number of finished free riders decreases with the arrival ratio increment in Fig. 2(b). The reason is that the increased arrival rate of the free riders produces more low priority bandwidth competition among themselves. Another notable issue is that the free rider could not get more benefit even though there is a higher arrival rate of general peers in the system as shown in Fig. 2(c) (the value of the finished peers' ratio keeps about 20, while the arrival ratio is 10). This can be explained by the assumption that general peers will immediately leave the system when they finish the downloading. 



Figure 2.(a) the number of general peers who complete their downloading jobs when the analysis arrives into a steady state. Figure 2.(b) the number of free riders who complete their downloading jobs when the analysis arrives into an steady state.

Figure 2.(c) the ratio of these finished peers to the finished free riders when the analysis arrives into a steady state.

The results indicate that the local private history-based credit strategy could effectively provide fairness for a single file exchange. A general peer could obtain a high-bandwidth reward to speed up its downloading process using credit exchange is expected, because it would re-access neighboring peers who build a credit relationship with it during the exchange process. In contrast, the free rider is just a beneficiary. Without any credit, it always belongs to the low priority queue with poor average uploading bandwidth.

\section{B. Experiment}

We employ an experimental study by using the test method in [7] [8]. Evaluating a P2P file sharing system like eMule in the real world is full of challenges. The evaluation tasks such as the entire system monitoring, collecting, and measuring are difficult to realize under the distributed and autonomous environment. In addition, the large number of online peers, the distinct behavior and motivations among different peers, the various client modifications of eMule's official version, and the sharing contents' popularity all limit the usability of current networking testbeds such as PlanetLab and network simulators like ns-2. However, whether or not the eMule network is fair can still be judged from the peer's viewpoint. For example, if a free rider obtained a similar download rate to that obtained by a general peer who followed the incentive strategy, it would be true that the system is unfair. Or, if the free rider's downloading bandwidth is limited compared with the general peer's, this would imply reasonable fairness. Our experiments focus on the downloading process of a single file, and the basic steps used to evaluate fairness are as follows:

1) Two peers are introduced into the eMule's Network: one is the general peer, and the other is the free rider. In our experiment, the free rider is an extremely malicious peer who will contribute nothing during its downloading process. The free rider is implemented by modifying one of eMule's popular client versions, eMule v0.49C. An unmodified client represents the general peer, which is running with eMule's recommended default configuration. The free rider and the general peer are running separately on two computers with the same configuration.
2) These two clients join the same file-swarm at the same time, and leave the system when the downloading job is completed. The uploading and downloading bandwidth are unlimited.

3) When the downloads are finished, the average download rate are calculated as:

$$
\text { Average Download Rate }=\frac{\text { File Size }}{\text { Download Time }}
$$

In order to keep the experimental results accurate, different types of contents with different file sizes are selected as the downloading resources, and after each test, the general peer's ID is randomly recreated to avoid the private history effect. The results are shown in Table 2:

Table 2: The downloaded file size, the average download and upload rate of the general peer and the free rider, and the ratio of the general peer's download rate to the free rider's download rate in different experiments

\begin{tabular}{|l|l|c|l|l|l|}
\hline \multirow{2}{*}{$\begin{array}{c}\text { File Size } \\
\text { (MB) }\end{array}$} & \multicolumn{3}{|c|}{$\begin{array}{c}\text { Average Download Rate } \\
\text { (KB/s) }\end{array}$} & \multicolumn{2}{c|}{$\begin{array}{c}\text { Average Upload Rate } \\
\text { (KB/s) }\end{array}$} \\
\cline { 2 - 6 } & $\begin{array}{c}\text { General } \\
\text { Peer }\end{array}$ & $\begin{array}{c}\text { Free } \\
\text { Rider }\end{array}$ & Ratio & General peer & Free Rider \\
\hline 114 & 34.34 & 14.69 & 2.34 & 264.86 & 0 \\
\hline 294 & 21.82 & 8.98 & 2.43 & 83.98 & 0 \\
\hline 524 & 25.57 & 2.22 & 11.52 & 240.50 & 0 \\
\hline 700 & 85.03 & 10.10 & 8.42 & 350.37 & 0 \\
\hline 1350 & 23.16 & 12.10 & 1.94 & 23.23 & 0 \\
\hline
\end{tabular}

The experimental results are greatly affected by the current number of seeds and peers in the system, the different upload and download bandwidth among a great number of general peers, and the obtained downloading bandwidth of free riders, etc. However, the results in Table 2 provide insight into the fairness policy of the eMule system, which emphasizes that the download rate of a free rider is always smaller than the general peer's. Even though real conditions are more complicated than the simple assumption of our mathematical model, the results still confirmed our model's conclusion that the local private-history based credit system of eMule can 




Figure 3. The number of peers in the queueing network. When using reward policy, general peer's number in the first several stations is reduced when comparing with Figure 4. Solid line: general peers; Dash line: free riders

provide fairness to the general peers and deal with free riders effectively. During the tests, the free rider's download rate was high at the beginning, and then reduced as its selfish behavior pushed itself into the low position of its neighbors' queues. Meanwhile, the general peer could continuously upload and benefit its download rate with the own credit. Thus, the free rider always obtained less average download rate than the general peer.

Sometimes the ratio of the download rate of the general peer to the free rider is not significant. The potential reason are: the system's capacity is much lager than the customer's request; plenty of general peers completing their jobs still stay in the system for contributing; or our free rider client's neighbors have higher upload rates than that of our general peer client's neighbors. Additionally, there remains an open question: how should one judge the fairness degree, which may relate the ratio of the general peer's upload rate to its download rate? This question will be addressed in our future work.

\section{Comparison with BitTorrent}

The popular peer-to-peer file sharing application "BitTorrent", whose "TFT" incentive algorithm was employed for fairness promotion, does not prohibit free riders from completing downloads. Free riders can still obtain enough bandwidth through two kinds of download channels:

1) free riders download from the seeds which only provide uploads and don't need to conform to the TFT strategy;

2) free riders download from the other peers without obeying the incentive policy, as each peer periodically unchokes its part of the upload slot to randomly chosen peers through the "optimistic unchoking" mechanism.

Research has shown that free riders of BitTorrent could receive higher download rates than the general peers in most common situations [8].

On the other hand, a peer's average download rate in eMule is totally decided by the position in the uploading queue of

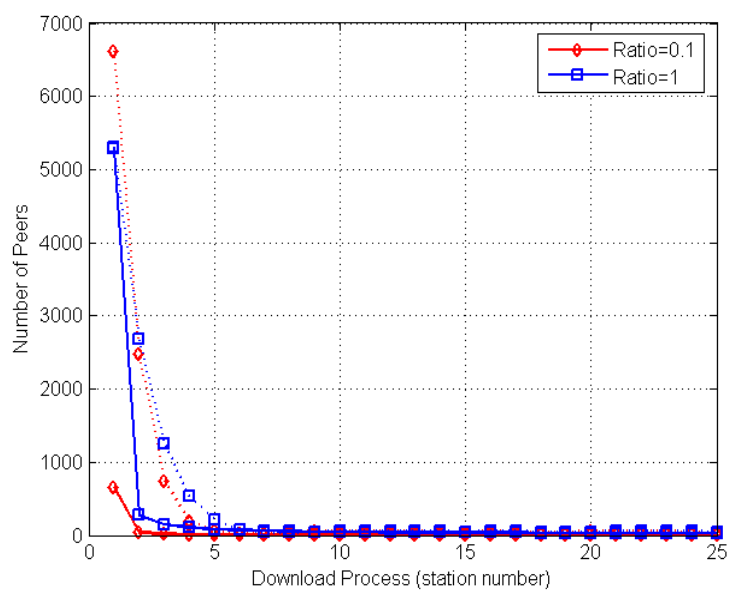

Figure 4. The number of peers in the queueing network. When using penalty policy, peers accumulated into the first several stations. Solid line: general peer; Dash line: free riders

uploaders. This credit-based rule does not only cover the general peers, but it theoretically covers the seeds in a long time period. Because the general peer always follow the credit-based incentive policy and thus get benefit through the credit, its average download rate could not be less than the free rider's. Even though the BitTorrent TFT incentive algorithm is rate-based, greedy, and its general peers may achieve higher download rate than eMule's, eMule's credit system could more fairly treat general peers and free riders from a fairness management perspective.

\section{NEWCOMER ISSUE AND SOLUTION}

\section{A. Newcomer Issue}

When a general peer has just arrived into the system, like a typical free rider without any content contributed to others, it is not easy to quickly receive its first chunks in the file swarm. This case is also called the first-chunk problem in [4] and is verified by the peer's U-shape distribution in the system [3]. Thus, there exists an inevitable fairness design issue about how to deal with a newcomer who may belong as either a general peer or a free rider. Generally, two opposite strategies are applied: one is penalizing all kinds of newcomers, while the other rewards newcomers. Our model is used to evaluate both situations under the steady state.

In Fig. 3, the general-peer and free rider populations at each service station with different arrival ratios are shown when all newcomers are awarded to the high priority queue through $P_{r}(0)=1$. Fig. 4 shows the similar setup except all newcomers are punished to the low priority queue by letting $P_{r}(0)=0$.

These graphs show that the credit system is still effective in dealing with free riders during one file exchange. In Fig. 3, free riders accumulate around the first several service stations even when rewarded with the high priority queue upon first arriving in the system. Likewise in Fig. 4, when the penalty strategy is employed, there is a prominent growth of the 
general peers' population at the first service station $S_{1}$ when the arrival ratio rises. The explanation is that after a general peer first arrives into the system, it has not owned any exchangeable content and has to stay in the low priority queue competing with free riders increment. If the reward policy is employed, general peers do not accumulate at the first service station and their downloading process will be sped up.

Current peer-to-peer file sharing applications tend to the reward solution: i.e., BitTorrent's "optimistic unchoking" scheme provides some bandwidth to a newcomer, even if this introduces unfairness into the system. eMule also adopts a similar policy that each newcomer will quickly receive a first chunk no matter which type of peer it belongs to.

\section{B. Long-time History Improvement}

At issue is the case when a free rider attempts to gain extra benefit by pretending to be a newcomer via regularly changing its ID. In this case, both the penalty and reward strategies for coping with the newcomer will not continue to keep the system fair. Furthermore, this newcomer issue could bring a Sybil attack [18] to damage the fairness of a P2P network that is based on the global reputation system. Thus, additional, complex authentication or a global reputation system based on public history is proposed. However, this may aggravate the network's burden due to the large information exchange, or it may require a management center as an addition to the traditional peer-to-peer structure.

The current eMule's fairness implementation is also vulnerable to the newcomer issue because of its reward policy to newcomers. Moreover, even though eMule uses an easy way to distinguish the general peer from the free rider by drawing support from the local private history-based credit, the evaluation results only show that this rule is useful during single file sharing, and we call this single file download period a short-time local private history. However, it is difficult for a general peer to use its rewarded credit across different file downloads. As an example, assume a peer has finished downloading a file, after which it leaves the system. Several hours later or several days later, it rejoins the system to download another file. This raises the question of whether its previous earned credit can be guaranteed to use for the current download process, especially in case of long term local private history. The answer is no, because when a general peer arrives as a newcomer, the probability of meeting its neighbors with whom there is an existing credit relationship, is close to zero. This also causes $P_{r}(0)$ in our analytic model to be almost zero. It is due to many uncertain factors, such as:

1) The large total number of peers in the system as well as the relative small number of this general peer's previous neighbors.

2) Whether its previous neighbors have desirable content?

3) Whether its neighbors are currently online?

4) Whether its neighbors want to share their content?

Hence, the performance of general peers and overall system fairness will be strengthened if eMule could increase the

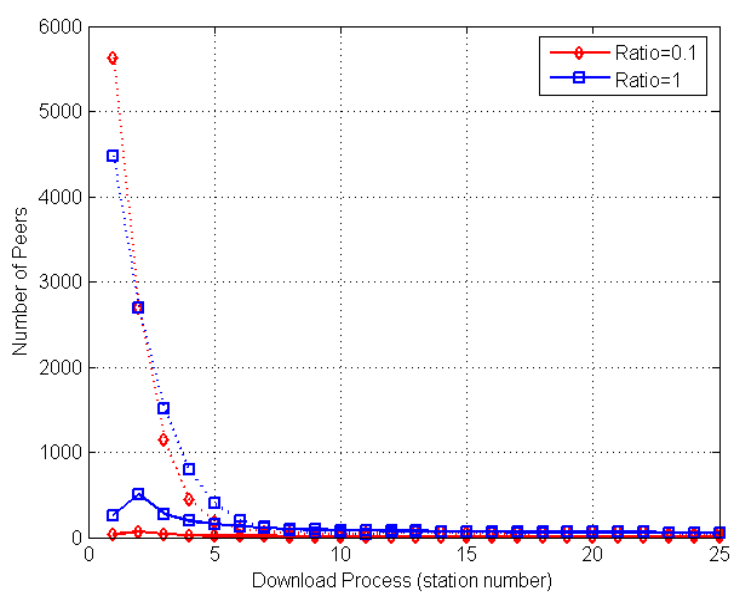

Figure 5. The number of peers in the queueing network. When using longtime local private history-based proposal. General peers don't accumulated into the first several stations, and free riders are still punished. Solid line: general peers; Dash line: free riders.

probability of newcomers meeting previous downloaders and continuously utilizing private credit history. This would also increase $P_{r}(0)$ in our mathematical model. The solution will be implemented as follows: During each file exchange process, every downloader not only provides credit to its direct uploader, but also gives some additional credit to those peers who have exchanged this file's content with the uploader. As the downloader not the uploader keeps the credit records [17], thus, more general peers besides the direct uploaders will gain future transaction benefit and an increased $P_{r}(0)$ can be guarantied.

The improvement is still local, private history-based, and simple. Instead of maintaining private credit between two peers, our method will let the credit operations cover more general peers with exponential growth, but it doesn't need to calculate a global credit score like the global reputation system. It will promote more sharing behaviors, because peers will take care of not only short-term behaviors, but their longterm generous behaviors. This could correspond to a higher reward probability during their first chunk downloads in the future. On the other hand, our solution following the credit policy does not provide any benefit to the free rider even if it only repeatedly changes its identity without uploading anything. Because general peers are more willing to share their contents to earn more incentive credit, the fairness will be strengthened and the system's capacity will be improved.

This proposal is also tested through the queueing network model. Fig. 5 provides numerical analysis results to support this idea. The results are shown with a small step of $P_{r}(0)=0.2$ for the general peer, which means the increasing probability of meeting previous neighbors if following our proposal. On the other hand, we still restrict the free rider's $P_{r}(0)=0$. In Fig. 5, the general peer's populations are very similar to the reward policy results shown in Fig. 3, while more free riders as newcomers are restrained into the first station comparing with Fig. 3. Thus, without losing the benefit of local private 
history-based credit, our solution can still improve the whole system's fairness as well as extend eMule's credit validity from short-term to long-term. Several design issues still exist such as determining the optimal quantity of the additional credit. We will focus on them in our future work.

\section{SUMMARY AND FUTURE WORK}

In this paper, we both develop a simple queueing network model and do experimental study for researching credit systems used in peer-to-peer file sharing networks such as eMule. The results show that this local-based credit scheme could effectively provide fairness for the system in the shorttime history. Our model also reveals the newcomer's fairness problem. After comparing with existing penalty and reward strategies, a simple, local, and long-term history-based scheme is suggested. This strategy not only rewards incoming general peers having good long-time history records, but it also restrains the benefit to free riders even though they repeatedly join the system using distinct IDs. Future work will aim to the credit's long history effect for system fairness. The experimental study will not restrained by one file downloading process, and the same peer ID will be maintained during consistent downloading over a long-term period. Another research ideas may include how the seeds affect the credit policy, and how to judge the fairness degree.

\section{ACKNOWLEDGMENT}

We would like to thank Dr. Caterina Scoglio, Phillip Schumm, and Mina Youssef for their comments.

\section{REFERENCES}

[1] Bram Cohen, "Incentives build robustness in BitTorrent" 1st Workshop on Economics of Peer-to-Peer Systems 2003.
[2] Runfang Zhou, Kai Hwang, "Gossip-based Reputation Aggregation for Unstructured Peer-to-Peer Networks" Parallel and Distributed Processing Symposium, 2007. IPDPS 2007.

[3] Ye Tian, Di Wu, Kam-Wing Ng, "Modeling, analysis and improvement for bitTorrent-like file sharing networks" in: INFOCOM 2006. 25th IEEE International Conference on Computer Communications.

[4] Minghong Lin, Bin Fan, John C.S. Lui, Dah-Ming Chiu, "Stochastic analysis of file-swarming systems" in the special issue of Performance Evaluation Journal, 64(9-12), 2007.

[5] Michal Feldman, Kevin Lai, Ion Stoica, John Chuang, "Robust incentive techniques for peer-to-peer networks" In Proceedings of the 5th ACM conference on Electronic commerce 2004.

[6] Chiranjeeb Buragohain, Divyakant Agrawal, Subhash Suri, "A game theoretic framework for incentives in $\mathrm{p} 2 \mathrm{p}$ systems" In Proceedings of the 3rd International Conference on Peer-to-Peer Computing 2003.

[7] Thomas Locher, Patrick Moor, Stefan Schmid, Roger Wattenhofer, "Free riding in BitTorrent is cheap" In Proceedings of HotNets 2006.

[8] M Sirivianos, JH Park, R Chen, X Yang, "Free-riding in BitTorrent networks with the large view exploit" - Proc. of IPTPS, 2007.

[9] Sepandar D. Kamvar, Mario T. Schlosser, Hector Garcia-Molina, "The Eigentrust algorithm for reputation management in P2P networks" Proceedings of the 12th international conference on World Wide Web ACM WWW'03.

[10] Chrysanthos Dellarocas, "Immunizing online reputation reporting systems against unfair ratings and discriminatory behavior" $r$, Proceedings of the 2nd ACM conference on Electronic commerce.

[11] Sanja Petrovic, Patrick Brown, "Fluid model for eMule file sharing system", In the Universal Multiservice Networks, 2007. ECUMN '07.

[12] Kenji Leibnitz, Tobias Hoßfeld, Naoki Wakamiya, and Masayuki Murata, "On pollution in eDonkey-like peer-to-peer file-sharing networks" In Proc. of GI/ITG MMB 2006

[13] Jia Yang, Hao Ma, Weijia Song, Jian Cui, Changling Zhou, "Crawling the eDonkey network", In proceeding of the fifth international conference on Grid and Cooperative Computing Workshops 2006

[14] Walid Saddi, Fabrice Guillemin, "Measurement based modeling of eDonkey peer-to-peer file sharing system" In "Managing Traffic Performance in Converged Networks", page 974-985 2007

[15] Slyck. http://www.slyck.com/.

[16] Bolch, Greiner, de Meer, Trivedi, Queueing Networks and Markov Chains Modeling and Performance Evaluation with Computer Science Applications Johe Wiley \& sons, 1998

[17] http://www.emule-project.net/.

[18] John Douceur, Judith S. Donath, "The sybil attack" In IPTPS 2002.

[19] http://en.wikipedia.org/wiki/Asymmetric_digital_subscriber_line. 